\title{
IMPOSTO SOBRE CIRCULAÇÃO DE MERCADORIAS E SERVIÇOS - ICMS SOCIOAMBIENTAL: AVALIAÇÃO DA POLÍTICA DO ESTADO DE PERNAMBUCO NOS ÚLTIMOS CINCO ANOS NA PERSPECTIVA DA GESTÃO AMBIENTAL.
}

\section{Felipe Luiz Lima De Paulo ${ }^{1}$}

Resumo: O Imposto sobre Circulação de Mercadorias e Serviços - ICMS Socioambiental é um instrumento de política pública adotado pelo Estado de Pernambuco com o objetivo de incentivar os governos municipais adotarem ações voltadas para a gestão ambiental e social. Assim, o objetivo deste artigo é de avaliar os resultados da política do ICMS Socioambiental nos últimos cinco anos nos municípios do Estado de Pernambuco para os critérios unidades de conservação e tratamento e destinação final de resíduos sólidos. Esta pesquisa justifica-se, pois ao final será possível construir uma base informacional para tomada de decisões para gestores dos municípios do Estado de Pernambuco e para aqueles que fazem a gestão do ICMS Socioambiental. A metodologia de pesquisa adotada foi baseada na análise da evolução dos Índices de Conservação de Biodiversidade dos Municípios - ICBM, na análise da evolução das pontuações para o tratamento e destinação final de resíduos sólidos e na análise dos recursos recebidos do ICMS em cada município para os critérios mencionados, comparando-os com as despesas realizadas nas funções urbanismo, saneamento e gestão ambiental. Ao final, observou-se que os indicadores analisados vão perdendo desempenho na medida em que a análise se desloca dos municípios da RMR ao sertão.

\footnotetext{
${ }^{1}$ felipe@uast.ufrpe.br
} 
Palavras-chave: ICMS Socioambiental. Índice de Conservação da Biodiversidade do Município - ICBM. Sistemas de Tratamento e Destinação Final de Resíduos Sólidos.

\section{INTRODUÇÃO}

O Imposto sobre Circulação de Mercadorias e Serviços - ICMS Socioambiental tem a proposta, em síntese, de promover o desenvolvimento sustentável no Estado de Pernambuco. Incluído nos instrumentos de políticas públicas do referido Estado, tal instrumento surgiu da necessidade de conter a degradação ambiental e de promover a melhoria dos indicadores sociais que refletem a qualidade de vida para a população atual e para as gerações futuras e tem como base o artigo 158 da Constituição Federal do Brasil.

Baseado na experiência de outros Estados brasileiros com o ICMS Ecológico ou ICMS Verde (denominações para o ICMS Socioambiental em outros Estados), como foi o exemplo do pioneiro Estado do Paraná que teve inicio na década de 90, o ICMS Socioambiental teve início com a Lei Estadual ㄲo 11.899, de 21 de dezembro de 2000 e hoje, após mudanças da legislação, entre outros aspectos, tem a proposta de incentivar os municípios a melhorarem as políticas públicas ambientais por meio da criação e manutenção das unidades de conservação e do adequado tratamento e disposição final de resíduos sólidos.

Justificando este estudo, basta ver, por exemplo, o trabalho de Pedrosa, Reis e Silva (2011) que aponta para a necessidade de políticas públicas mais efetivas para os municípios do Estado de Pernambuco. Um exemplo é município de Paulista-PE, que apresentou um aumento das degradações ambientais refletidas pelo Índice das Pressões Antrópicas (IPA) que, em 2002 era de 0,909 e, em 2006, 0,535². Dessa forma, ao final desta pesquisa, o gestor municipal e o gestor da política do ICMS Socioambiental terão uma base de dados informacional que dará condições aos mesmos de tomar decisões para melhoria deste instrumento de política pública.

\footnotetext{
${ }^{2}$ Elaboração: grupo de pesquisa do NUPESP. Sobre o cálculo dos índices, ver PEDROSA, REIS e SILVA, 2011
} 
Assim, este estudo tem como objetivo principal o de avaliar os resultados da política do ICMS Socioambiental nos últimos cinco anos nos municípios do Estado de Pernambuco para os critérios unidades de conservação e tratamento e destinação final de resíduos sólidos. Este recorte analítico dos critérios foi feito para que a pesquisa pudesse ser focada apenas no desempenho da gestão ambiental dos municípios do Estado.

\section{REFERENCIAL TEÓRICO}

O Imposto sobre Circulação de Mercadorias e Serviços Intermunicipal e Interestadual e de Comunicação - ICMS é uma espécie de tributo. Tributo, conforme o Código Tributário Nacional - CTN (Lei 5.172, de 25 de Outubro de 1966) é assim definido:

Art. 3‥ Tributo é toda prestação pecuniária compulsória, em moeda ou cujo valor nela se possa exprimir, que não constitua sanção de ato ilícito, instituída em lei e cobrada mediante atividade administrativa plenamente vinculada.

Em outras palavras, pode-se dizer que o tributo é um valor pago para o Estado, somente pode ser criado por meio de lei (e o mesmo constitui dever), e só podem ser cobrados pela União, Estados, Municípios e Distrito Federal. Ainda em relação aos conceitos básicos de tributos, à luz do Código Tributário Nacional - CTN, existem três espécies distintas, sendo elas: Impostos, Taxas e Contribuição de Melhoria (ARTIGO $5^{\circ}$ do CTN).

O tributo pode ser instituído com caráter estritamente arrecadatório para financiar as atividades do setor público (fiscalidade), como também o tributo pode ser utilizado para regular o comportamento social, estimulando ou desestimulando esse comportamento (extrafiscalidade). Um exemplo clássico é o caso do Imposto sobre Produtos Industrializados - IPI, um tributo que tem a função de arrecadar recursos financeiros para os cofres públicos, mas que também pode estimular ou desestimular o consumo de bens na sociedade, ou seja, por exemplo, quando o governo tem interesse em estimular o consumo de veículos novos, diminui-se a alíquota cobrada (percentual aplicado sobre a 
base de cálculo do imposto), e quando o governo tem interesse em desestimular o consumo, como é o caso de cigarros e bebidas alcoólicas, aumenta-se a alíquota cobrada.

Em relação à competência tributária, pode-se dizer que não envolve somente o poder de fiscalizar e cobrar tributos, mas também o de legislar a respeito. Observe-se que não tem competência tributária o ente público desprovido do poder legislativo. Dessa forma, a competência tributária pertence à União, aos Estados, ao Distrito Federal e aos Municípios. Sob esta ótica, a Constituição Federal do Brasil de 1988 estabeleceu a competência de cada esfera federativa para cada tributo. Exemplo disso é o artigo 155 da CF/88 que estabelece os tributos pertencentes aos estados e distrito federal (Imposto sobre Circulação de Mercadorias e Serviços, e o Imposto sobre Propriedade de Veículo Automotor, por exemplos).

A Constituição Federal de 1988, artigo 158, também define regras para repartição das receitas tributárias entre os municípios. O artigo define, por exemplo, regras para repartição do produto arrecadado do ICMS para os municípios. Pode-se dizer que $25 \%$ do produto arrecadado do ICMS pelos Estados devem ser repassados para os municípios. O gráfico 1 abaixo ilustra bem como deve ser repassado o tributo para os municípios em cada Estado:

Gráfico 1 - REPARTIÇÃO DAS RECEITAS TRIBUTÁRIAS - ICMS (CF/88, art. 158)

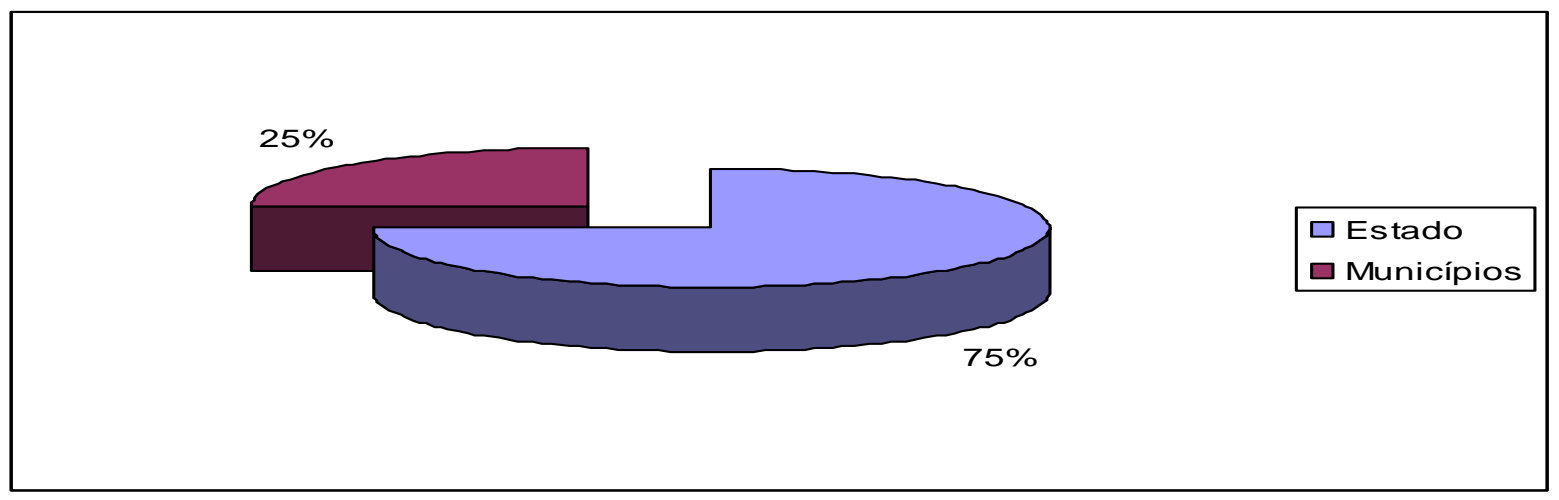

Fonte: $\mathrm{O}$ autor. 
Já em relação aos critérios de repasse aos municípios, o dispositivo legal também determina que $75 \%$ da quantia que pertence aos municípios devem ser repassados por meio do valor adicionado fiscal, ficando 25\% livre para cada Estado definir, por meio de legislação própria, os critérios de repasse para os municípios. O gráfico 2 abaixo ilustra bem como funciona esta repartição:

\section{Gráfico 2 - REPARTIC̣ÃO DAS RECEITAS TRIBUTÁRIAS - ICMS}

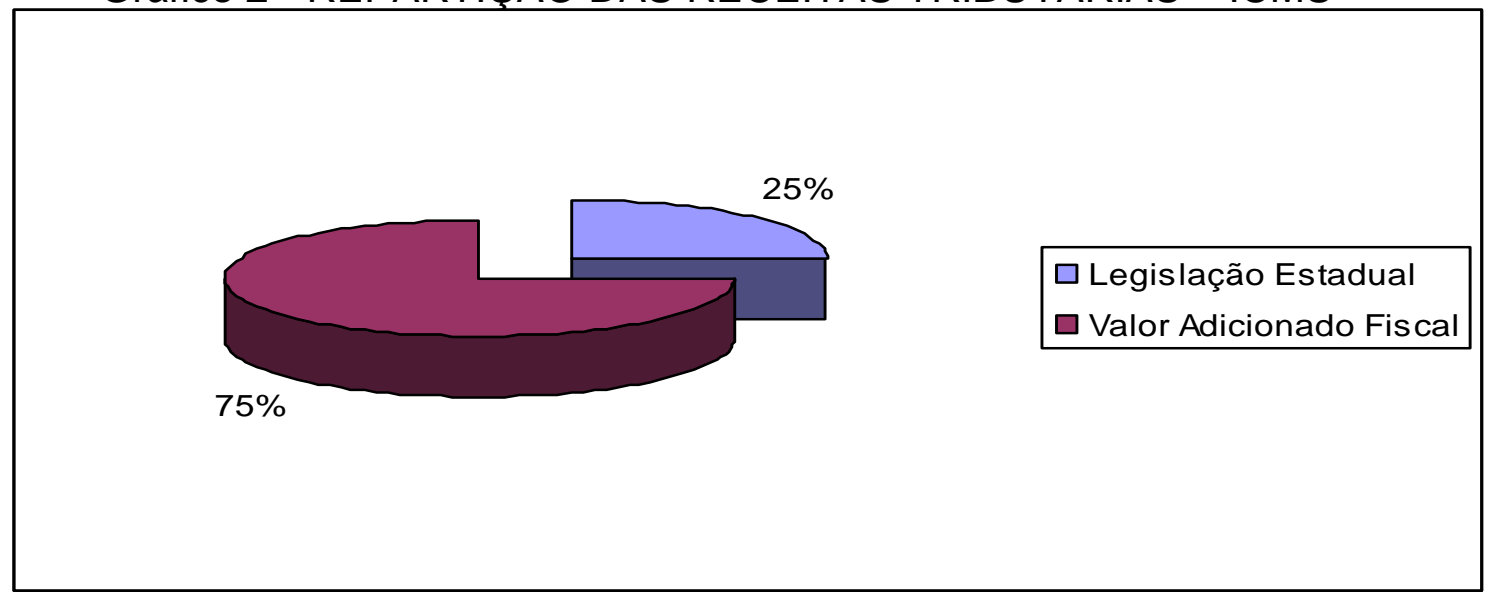

Fonte: $\mathrm{O}$ autor.

Dessa forma, cada Estado pode definir critérios que possam estar atrelados com suas políticas públicas para a melhoria de qualidade de vida da população. O ICMS Socioambiental foi criado nesta perspectiva.

\section{O ICMS SOCIOAMBIENTAL NO ESTADO DE PERNAMBUCO}

O ICMS Socioambiental se baseia no artigo 158 da Constituição Federal do Brasil de 1988, quando permite que cada Estado possa adotar critérios específicos para o repasse do ICMS para os municípios. O Estado do Paraná, por exemplo, foi o pioneiro em adotar critérios ambientais para o repasse do ICMS, estimulando a melhoria das condições ambientais no Estado. No Brasil, as adoções de critérios ambientais para essa parcela destinada aos municípios em diversos Estados ganharam nomes diferentes, tais como 
ICMS Ecológico, ICMS Verde e ICMS Socioambiental (no caso do Estado de Pernambuco).

O termo ICMS Socioambiental foi adotado no Estado de Pernambuco devido ao fato de contemplar vários critérios socioambientais para o repasse do ICMS aos municípios. São levados em consideração, por exemplo, a participação relativa de cada município ${ }^{3}$, unidades de conservação, sistemas de tratamento ou de destinação final de resíduos sólidos, mortalidade infantil, programa de saúde da família, educação, receita tributária própria, PIB "per capita", número de crimes violentos letais intencionais, presídios e penitenciárias e população do município. O gráfico 3 demonstra como tais critérios são distribuídos no percentual de $25 \%$ que a constituição federal permite que o Estado trabalhe por meio de legislação específica aos municípios:

\section{Gráfico 3 - DISTRIBUIÇÃO DOS CRITÉRIOS DO ICMS SOCIOAMBIENTAL}

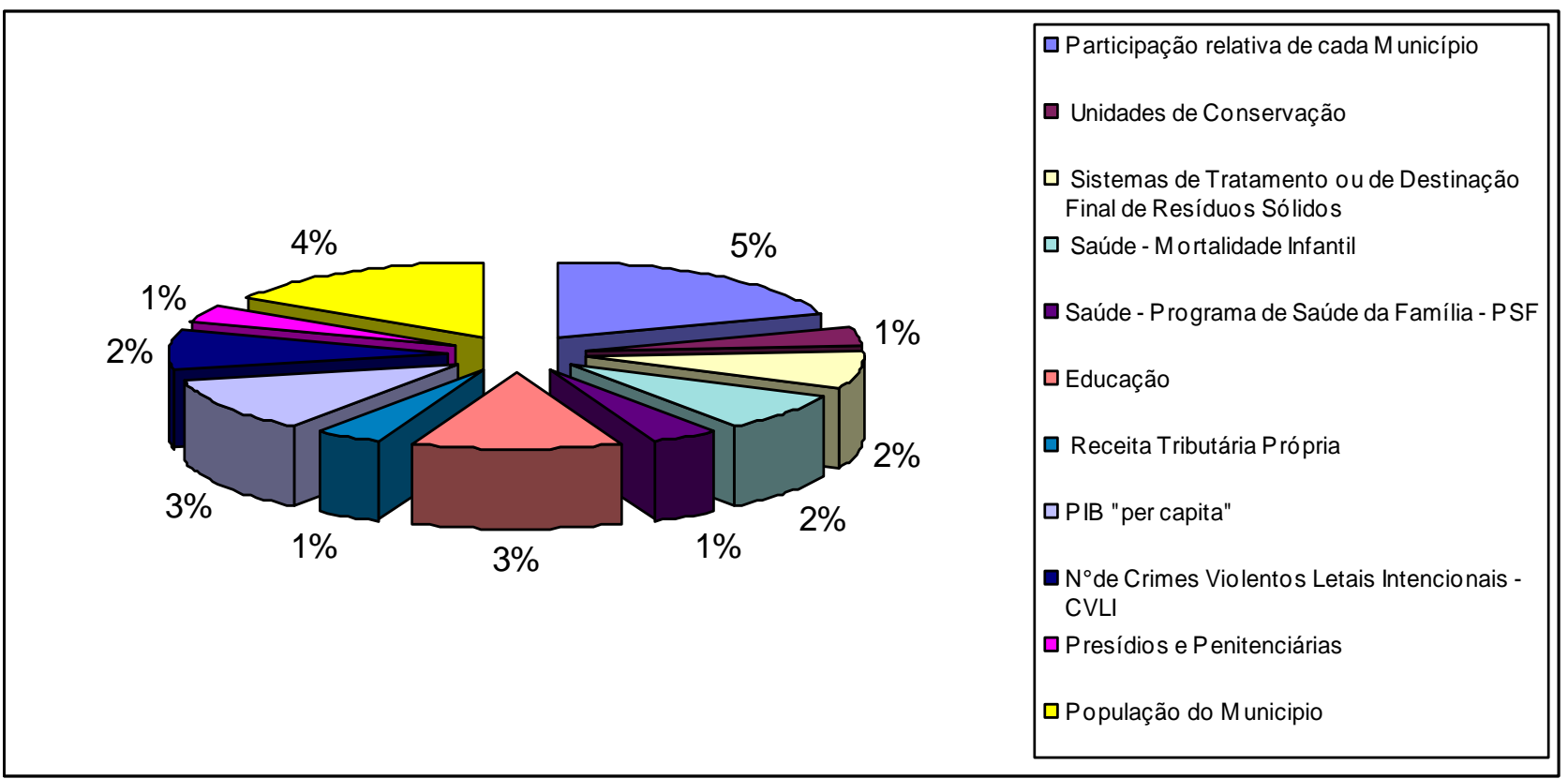

Fonte: os autores. Lei n 13.368, de 14 de Dezembro de 2007.

\footnotetext{
3 " $25 \%$ (vinte e cinco por cento) de participação relativa no somatório das diferenças entre o índice percentual de participação vigente para cada Município no exercício anterior e a percentagem determinada nos termos do inciso I". Esse mecanismo foi criado, na época, para reduzir os impactos, sobre o índice e, consequentemente, sobre as transferências, das grandes variações no valor adicionado fiscal, sujeito à precariedade das estatísticas apuradas na atividade arrecadadora.
} 
Estes critérios já passaram por diversas mudanças devidas justamente ao efeito redistributivo característico do ICMS Socioambiental, ou seja, ocasiona um efeito "hobin hood" tirando de uns municípios e contemplando outros mediante adequações à legislação do ICMS Socioambiental. Jorge Jatobá, então secretário da fazenda do Estado de Pernambuco na época, relatou em seu estudo publicado na CEPAL (Comisión económica para América latina y el Caribe) que na época houve diversas reclamações na Associação Municipalista do Estado de Pernambuco - AMUPE o que ocasionou mudanças nos percentuais e nos critérios adotados no ICMS Socioambiental desde sua criação no ano 2000 (JATOBÁ, 2005, p. 154).

O fato é que o ICMS Socioambiental pode ser entendido como um instrumento econômico de gestão ambiental utilizado pelo governo estadual para que os municípios aperfeiçoem as políticas socioambientais. Trata-se de um estímulo financeiro para que os governos municipais promovam o desenvolvimento sustentável, como por exemplo, por meio da criação e manutenção de unidades de conservação e de adequados sistemas de tratamento e disposição final de resíduos sólidos.

Assim, pode-se observar que o ICMS Socioambiental sendo utilizado como um instrumento de política pública, como já foi mencionado, estimula benefícios por não ocasionar queda na arrecadação tributária, diferente do IPI (já mencionado), e por trabalhar como um instrumento econômico de gestão ambiental baseado no princípio do "protetor recebedor", diferente de políticas ambientais já adotadas que tem como base o princípio do "poluidor pagador". Sabe-se que a disposição a receber é maior que a disposição a pagar (HEMPEL, 2006).

\section{METODOLOGIA UTILIZADA}

Esta pesquisa abordou dois critérios de repasse do ICMS Sociambiental para os municípios: unidades de conservação e resíduos sólidos. Esta escolha foi definida justamente pelo objetivo proposto no artigo, que é de avaliar os resultados da política do ICMS Socioambiental nos últimos cinco anos nos municípios do Estado de Pernambuco 
para os critérios unidades de conservação e tratamento e destinação final de resíduos sólidos, ou seja, focando na perspectiva da gestão ambiental. Dessa forma, ao final da pesquisa, será possível mapear a evolução das unidades de conservação no Estado de Pernambuco, bem como a evolução dos sistemas de tratamento e destinação final de resíduos sólidos. Com isso, será possível sugerir melhorias no instrumento de política pública denominado ICMS Socioambiental, bem como demonstrar a importância deste incentivo financeiro para as finanças públicas municipais.

Para avaliar a evolução das unidades de conservação, utilizou-se o Índice de Conservação da Biodiversidade do Município - ICBM. Logo abaixo segue a metodologia de calculo do indicador que é utilizada pela Agência Estadual de Meio Ambiente e Recursos Hídricos - CPRH:

$$
\begin{aligned}
& \mathrm{CB}=(\mathrm{AUC} / \mathrm{AM} \times \mathrm{FC}) \times \mathrm{AQUC} \\
& \mathrm{ICBM}=(\mathrm{CBM} / \mathrm{CBE}) \times 100
\end{aligned}
$$

a) CB - Coeficiente de Conservação da Biodiversidade da Unidade de Conservação.

b)CBM - Somatório de todos os Coeficientes de Conservação da Biodiversidade calculados para o Município;

c) CBE - Somatório de todos os Coeficientes de Conservação da Biodiversidade calculados para todos os Municípios do Estado. (Decreto noㅡ 25.574, de 25 de Junho de 2003)

Os resultados deste indicador foram obtidos por meio de portarias disponíveis no site da Secretaria da Fazenda do Estado de Pernambuco - SEFAZ-PE. Complementarmente, foram obtidos dados dos percentuais de repasse do ICMS Socioambiental por município pela SEFAZ-PE por meio de ofício enviado pelos autores. Também foram obtidos os nomes de todas as unidades de conservação do Estado de Pernambuco.

Já para avaliar a evolução dos sistemas de tratamento e destinação final de resíduos sólidos, foram analisadas as pontuações utilizadas em cada estágio de evolução dos sistemas de tratamento, que são dois, sendo eles: unidades de compostagem e aterro 
sanitário como seguem (informações da Secretaria da Fazenda do Estado de Pernambuco - SEFAZ-PE):

\footnotetext{
Unidade de compostagem:

licença prévia: 2 pontos; licença de implantação: 3 pontos; licença de operação; 5 pontos; operação regular: 10 pontos.

Aterro sanitário:

licença prévia: 3 pontos; licença de instalação: 7 pontos; licença de operação; 10 pontos; operação regular: 40 pontos; desativação do vazadouro - lixão: 20 pontos.
}

Os resultados destes indicadores, bem como a localização de todos os sistemas de tratamento e destinação final de resíduos sólidos no Estado de Pernambuco e os percentuais de repasse para cada município que irão ser úteis para o cálculo do repasse financeiro do ICMS Socioambiental para este critério, também foram obtidos por meio de portarias da Secretaria da Fazenda do Estado de Pernambuco - SEFAZ/PE e por meio de ofício enviado pelos autores.

Complementarmente, foram obtidos os dados contábeis do ano 2010 (são os dados mais recentes encontrados na base de dados utilizados) das despesas nas funções e subfunções que tratam da gestão ambiental de cada município do Estado de Pernambuco. Estes dados contábeis foram disponibilizados pela Secretaria do Tesouro Nacional por meio de seu endereço eletrônico, mais conhecido como FIBRA - Finanças do Brasil. Estes dados foram importantes para verificar se os recursos financeiros obtidos por meio do ICMS Socioambiental estão sendo gastos proporcionalmente nas funções relacionadas com a gestão ambiental que são: gestão ambiental, saneamento e urbanismo. Sabe-se, contudo, que não há qualquer obrigação do governo municipal em realizar estes gastos, pois não há qualquer vinculação da transferência do ICMS aos municípios com despesas em funções específicas.

O período de análise dos dados correspondeu aos últimos cinco anos (2008 a 2012) e contemplou todos os municípios do Estado de Pernambuco, organizados mediante as mesorregiões geográficas. 


\section{RESULTADOS DA PESQUISA}

Os resultados a serem apresentados a seguir demonstram a evolução do tratamento dado pela legislação estadual de Pernambuco às unidades de conservação bem como demonstram a evolução do tratamento dado pela legislação estadual de Pernambuco aos sistemas de tratamento e destinação final de resíduos sólidos. O período de análise correspondeu aos últimos cinco anos (2008 ao ano 2012), conforme descrito no capítulo da metodologia.

\subsection{EVOLUÇÃo do tRATAMENTO DADO PELA LEGISLAÇÃo ESTAdUAL DE PERNAMBUCO ÀS UNIDADES DE CONSERVAÇÃO -UC}

Analisando o desempenho do Índice de Conservação da Biodiversidade dos Municípios - ICBM nos municípios da Região Metropolitana do Recife, observa-se que a metade dos municípios apresentou aumento no indicador, exceto o município de Cabo de Santo Agostinho, Igarassu, Ipojuca, Jaboatão dos Guararapes, Moreno, Paulista e São Lourenço da Mata.

Estes indicadores refletem, com base nos dados atualizados para o ano 2010, as seguintes unidades de conservação: APA Aldeia-Beberibe, APA Estuário Rio Timbó, ZCVS APA Aldeia - Beberibe (Reserva Ecológica Mata de Miritiba), Reserva Ecológica Mata de São Bento, todas localizadas no município de Abreu e Lima; APA Estuário dos Rios Jaboatão e Pirapama, Reserva Ecológica Mata Serra do Cotovelo, Reserva Ecológica Mata de Bom Jardim, Reserva Ecológica Mata de Contra Açude, Reserva Ecológica Mata de Duas Lagoas, Reserva Ecológica Mata do Camaçari, Reserva Ecológica Mata do Cumaru, Reserva Ecológica Mata do Sistema Gurjaú, Reserva Ecológica Mata do Urucu, Reserva Ecológica Mata do Zumbi, todas localizadas no município do Cabo de Santo Agostinho; APA - Aldeia-Beberibe (Demais Zonas), localizada no município de Camaragibe; APA Estuarina do Rio Sirinhaém e Maracaípe, APA de Sirinhaem (ZCVS), RPPN Nossa Senhora do Oiteiro de Maracapipe, todas localizadas no município de 
Ipojuca; APA de Santa Cruz (demais zonas), ZPVS da APA de Santa Cruz (APA Estuário do Canal de Santa Cruz), ZPVS da APA de Santa Cruz (APA Estuário do Rio Jaguaribe), Refúgio de Vida Silvestre Mata de Jaguaribe, Refugio de Vida Silvestre Mata de Santa Cruz, Refúgio de Vida Silvestre Mata do Amparo, Refúgio de Vida Silvestre Mata Engenho Macaxeira, Refúgio de Vida Silvestre Mata do Engenho São João, Refúgio de Vida Silvestre Mato Lanço dos Cações, todas localizadas no município de Itamaracá; APA Estuário dos Rios Jaboatão e Pairapama, Reserva Ecológica Mata de Jangatinha, Reserva Ecológica Mata de Manassu, Reserva Ecológica Mata de Mussaíba, Reserva Ecológica Mata do Engenho Salgadinho, Reserva Ecológica Mata do Sistema Gurjaú, RPPN Sta. Beatriz de Carnijó, todas localizadas no município de Jaboatão dos Guararapes; RPPN Sta;. Beatriz de Carnijó, Reserva Ecológica Mata do Cumaru, Reserva Ecológica Mata de Caraúna, Reserva Ecológica Mata do Eng. Moreninho, Reserva Ecológica Mata do Sistema Gurjaú, Reserva Ecológica Mata Serra do Cotovelo, todas localizadas no município de Moreno; Reserva Ecológica Mata do Passarinho, ARIE Horto Del Rey, ARIE Mangue de Santa Teresa, todas localizadas no município de Olinda; APA Estuário do Rio Timbó, Estação Ecológica de Caetés, Reserva Ecológica Mata de Jaguarana, APA AldeiaBeberibe (Demais Zonas), Reserva Ecológica Mata do Janga, todas localizadas no município de Paulista; APA do Eng. Uchoa (demais zonas), APA Aldeia-Beberibe (Demais zonas), ZPVS APA Eng. Uchoa, Parque Estadual de Dois Irmãos, Reserva Ecológica Mata de Dois Unidos, Reserva Ecológica Mata do Curado, Reserva Ecológica Mata do Jardim Botânico, Reserva Ecológica Mata do São João da Várzea, todas localizadas no município de Recife; Estação Ecológica do Tapacurá, Reserva Ecológica Mata do Outeiro do Pedro, APA Aldeia-Beberibe (Demais Zonas), ZCVS da APA Aldeia-Beberibe (Reserva Ecológica Mata do Quizanga), Reserva Ecológica Mata do Tapacurá, todas localizadas no município de São Lourenço da Mata; APA Aldeia-beberibe (demais zonas), localizada no município de Araçoiaba; APA de SANTA Cruz (demais zonas), ZPVS da APA de SANTA Cruz (APA Estuário do CANAL DE Santa Cruz), todas localizadas no município de Itapissuma; APA Estuário do Canal de Santa Cruz, APA Estuário do Rio Timbó, APA Aldeia - Beberibe (Demais Zonas) , ZCVS da APA - Beberibe (Reserva Ecológica Mata da Usina São José), 
APA Nova Cruz (ZCVS I - APA Est. Canal de Santa Cruz), APA Nova Cruz (ZCVS II APA Est. Do Rio Timbó), APA Nova Cruz (ZCVS III), APA Nova Cruz (demais zonas), todas localizadas no município de Igarassu.

Já em relação aos municípios localizados na região da Zona da Mata, dos quatorze municípios que apresentaram pontuação para o critério "unidades de conservação", foi observado que apenas os municípios de Goiana, Maraial e Paudalho apresentaram evolução no indicador.

Estes indicadores refletem, com base nos dados atualizados para o ano 2010, as seguintes unidades de conservação: RPPN Laje Bonita, localizada no município de Quipapá; APA de Guadalupe (demais zonas); APA de Sirinhaém (demais zonas); REBIO Saltinho, todas localizadas no município de Rio Formoso; APA Estuário do Rio Uma, localizada no município de São José da Coroa Grande; APA Estuarina do Rio Sirinhaém e Maracaípe, APA de Guadalupe (demais zonas), APA de Sirinhaém (ZCVS), APA de Sirinhaém (demais zonas), todas localizadas no município de Sirinhaém; Parque Natural Municipal do Forte de Tamandaré, APA de Guadalupe (demais zonas), REBIO Saltinho, todas localizadas no município de Tamandaré; Reserva Ecológica Mata do Urucu, localizada no município de Vitória de Santo Antão; RPPN Fazenda Santa Rita, localizada no município de Água Preta; APA de Guadalupe, APA Estuário do Rio Uma, localizadas no município de Barreiros; RPPN Bicho Homem, RPPN Jussaral, localizadas no município de Catende; Reserva Ecológica Mata do Urucu, localizada no município de Escada; APA de Santa Cruz (demais zonas), ZPVS APA de Santa Cruz (APA Estuário do Canal de Santa Cruz), APA Estuário do Rio Goiana e Megaó, ZPVS da APA de Santa Cruz (APA Estuário do Rio Itapessoca), RESEX Acaú Goiana, RPPN Fazenda Tabatinga, todas localizadas no município de Goiana; RPPN Frei Caneca, localizada no município de Jaqueira; RPPN Contestado, localizada no município de Maraial, APA Aldeia-Beberibe (demais zonas), localizada no município de Paudalho.

Já em relação aos municípios localizados na região do Agreste, dos onze municípios que apresentaram pontuação para o critério "unidades de conservação", 
observa-se que apenas dois apresentou aumento no na pontuação do ICBM durante o período analisado, sendo eles: Lajedo e Venturosa.

Estes indicadores refletem, com base nos dados atualizados para o ano 2010, as seguintes unidades de conservação: Monumento Natural Parque Municipal Pedra Furada, localizada no município de Venturosa; Parque Nacional do Catimbau, localizada no município de Tupanatinga; RPPN Pedra do Cachorro, localizada no município de São Caetano; RPPN Brejo, localizada no município de Saloá; RPPN Reserva Calaça, localizada no município de Lajedo; Reserva Biológica de Pedra Talhada, localizada no município de Lagoa do Ouro; Parque Natural Municipal Professor João Vasconcelos Sobrinho, localizada no município de Caruaru; RPPN Fazenda do Catimbau, localizada no município de Buique; RPPN Fazenda Bituri, localizada no município de Brejo da Madre de Deus; Parque Ecológico de Serra Negra, localizada no município de Bezerros; RPPN Reserva Cabanas, localizada no município de Altinho.

Já em relação aos municípios localizados na região do Sertão, dos nove municípios que apresentaram pontuação no critério "unidades de conservação", apenas o município de Serrita apresentou aumento na pontuação durante o período analisado.

Estes indicadores refletem, com base nos dados coletados para o ano 2010, as seguintes unidades de conservação: FLONA de Negreiros, APA Chapada do Araripe, todas localizadas no município de Serrita; APA Cahapada do Araripe, localizada no município de Ipubi; Parque Nacional do Catimbau, localizada no município de Ibimirim; RPPN Maurício DANTAS, localizada no município de Betânia; RPPN Mauricio DANTAS, Reserva Biológica de Serra Negra, RPPN Cantidiano Valgueiro, localizadas no município de Floresta; APA Chapada do Araripe, localizada no município de Exu; APA Chapada do Araripe, localizada no município de Cedro; APA Chapada do Araripe, localizada no município de Araripina; APA Chapada do Araripe, localizada no município de Bodocó.

Por fim, observa-se que a região que apresentou, até o momento, incremento de unidades de conservação em um ritmo mais acelerado que as demais foi a Região Metropolitana do Recife - RMR. Contudo, há de se observar que estes indicadores refletem 
apenas um incremento em um ritmo mais acelerado que os demais municípios, não significando que o município deixou de ter unidades de conservação em seu território (as que já existiam). Outra observação que deve ser feita é que a avaliação qualitativa ainda não está sendo feita pela Agência Estadual de Meio Ambiente e Recursos Hídricos $\mathrm{CPRH}$, o que prejudica avaliar a qualidade das unidades de conservação.

\subsection{EVOLUÇÃo do tRATAMENTO DADO PELA LEGISLAÇÃO ESTADUAL DE PERNAMBUCO AOS SISTEMAS DE TRATAMENTO E DESTINAÇÃO FINAL DE RESÍDUOS SÓLIDOS}

Ao analisar a evolução do tratamento dado pela legislação do Estado de Pernambuco aos sistemas de tratamento e destinação final de resíduos sólidos, observase que a maioria dos municípios não apresentou nenhuma pontuação para o critério. Apenas 12 municípios apresentaram evolução para o critério analisado (7 da RMR, 4 da Zona da Mata e 1 do Agreste), sendo que os municípios de Araripina e Serrita, localizados no Sertão do Estado de Pernambuco, apresentaram reduções em suas pontuações ao ponto de não obter nenhum ponto para o critério ao final do período analisado.

Estas reduções demonstram diferente do critério "unidades de conservação", perda de algum estágio dos sistemas de tratamento e destinação de resíduos sólidos, ou seja, uma perda qualitativa, sendo considerado sem nenhum tratamento adequado de resíduos sólidos aquele município que não apresenta pontuação.

\subsection{PERFIL DAS DESPESAS MUNICIPAIS COM O MEIO AMBIENTE VERSUS RECURSOS DO ICMS SOCIOAMBIENTAL}

Neste tópico serão analisados os valores recebidos pelos municípios do Estado de Pernambuco para os critérios resíduos sólidos e unidades de conservação, comparando-os com as despesas nas funções saneamento, urbanismo e gestão ambiental (dados referentes ao ano 2010). Os dados têm origem em fontes secundárias, originados da 
Secretaria da Fazenda do Estado de Pernambuco - SEFAZ-PE e da Secretaria do Tesouro Nacional - STN por meio dos dados contábeis disponíveis conhecidos como FINBRA - Finanças do Brasil.

Logo abaixo, na tabela 1, estão discriminados apenas os municípios que receberam transferências do ICMS Socioambiental para o critério resíduo sólidos no ano 2010, sendo excluídos aqueles que não tiveram valores recebidos, ou em outras palavras, não alcançaram nenhuma pontuação para o critério.

Tabela 1 - ICMS Socioambiental para o critério "Resíduos Sólidos" versus despesas nas funções saneamento e urbanismo para o ano 2010

\begin{tabular}{l|c|c|c}
\hline \multicolumn{1}{c|}{ MUNICIPIOS } & $\begin{array}{c}\text { Valor dos } \\
\text { Resíduos }\end{array}$ & $\begin{array}{c}\text { DESPESA } \\
\text { SANEAMENTO }\end{array}$ & $\begin{array}{c}\text { DESPESA } \\
\text { URBANISMO }\end{array}$ \\
\hline AFRÂNIO & $9.888,39$ & $68.740,01$ & $1.143 .628,90$ \\
\hline AGRESTINA & $331.261,19$ & $786.845,74$ & $2.292 .886,52$ \\
\hline ALAGOINHA & $206.008,20$ & $449.451,83$ & $949.276,92$ \\
\hline ALTINHO & $365.870,57$ & NÃO DISPONIVEL & NÃO DISPONIVEL \\
\hline ARARIPINA & $1.170 .126,59$ & $6.102,51$ & $7.592 .740,85$ \\
\hline ARCOVERDE & $1.016 .856,49$ & $851.418,82$ & $8.459 .118,49$ \\
\hline BELO JARDIM & $1.133 .869,15$ & $93.140,47$ & $7.221 .480,56$ \\
\hline CABO SANTO AGOSTINHO & $2.523 .188,46$ & $938.772,88$ & $29.135 .033,03$ \\
\hline CARUARU & $2.093 .043,34$ & $1.635 .209,35$ & $32.839 .297,33$ \\
\hline ESCADA & $118.660,72$ & - & $6.799 .711,85$ \\
\hline FREI MIGUELINHO & $8.240,33$ & $23.473,90$ & $92.541,49$ \\
\hline GARANHUNS & $1.943 .069,36$ & $283.729,90$ & $21.411 .868,25$ \\
\hline GOIANA & $1.175 .070,79$ & - & $17.754 .971,79$ \\
\hline GRAVATÁ & $1.109 .148,16$ & - & $4.705 .782,49$ \\
\hline IATI & $291.707,61$ & $1.081 .440,30$ & $2.894 .987,26$ \\
\hline IBIMIRIM & $14.832,59$ & - & $3.788 .456,62$ \\
\hline IGARASSU & $1.358 .006,07$ & - & $7.921 .114,43$ \\
\hline JABOATÁO GUARARAPES & $9.595 .038,03$ & $4.555 .064,80$ & $71.729 .850,41$ \\
\hline LAJEDO & $530.677,13$ & $358.636,42$ & $2.043 .666,25$ \\
\hline OLINDA & $1.517 .868,43$ & $1.647 .492,85$ & $85.196 .907,94$ \\
\hline OROCÓ & $6.592,26$ & $825.908,54$ & $973.830,40$ \\
\hline PESQUEIRA & $952.581,93$ & $119.889,82$ & $5.634 .178,19$ \\
\hline PETROLÄNDIA & $451.569,98$ & $459.644,52$ & $7.016 .823,98$ \\
\hline RECIFE & $2.935 .204,87$ & $28.455 .344,30$ & $368.427 .920,71$ \\
\hline SAIRÉ & $225.784,99$ & $1.136 .637,07$ & $859.504,78$ \\
\hline SALGUEIRO & $850.401,86$ & $468.740,04$ & $7.060 .974,18$ \\
\hline STA. CRUZ DO CAPIBARIBE & $974.006,78$ & $325.194,00$ & $8.324 .281,26$ \\
\hline STA .MARIA DO CAMBUCÁ & $6.592,26$ & - & $906.975,15$ \\
\hline SÁO J. DA COROA GRANDE & $28.017,12$ & - & $3.219 .030,46$ \\
\hline TAQUARITINGA DO NORTE & $11.536,46$ & - & \\
\hline & & & \\
\hline
\end{tabular}


VERTENTES TOTAL DO ESTADO

$9.888,39$ $32.964 .608,50$

$1.938 .076,82$ $2.732 .477,56$

Fonte: Elaboração dos autores com base nos dados da SEFAZ-PE e do FINBRA

Por meio da análise da tabela 1, com exceção do município de Altinho que não teve os dados contábeis disponibilizados, é possível observar que todos os municípios apresentaram despesas em pelo menos uma das funções estudadas. Observe-se também que todos apresentaram despesas em pelo menos uma das funções estudadas que superaram os valores recebidos pelo ICMS Socioambiental no critério resíduos sólidos.

Já na tabela 2 estão discriminados apenas os municípios que receberam transferências do ICMS Socioambiental para o critério unidades de conservação no ano 2010, sendo excluídos aqueles que não tiveram valores recebidos, ou em outras palavras, não alcançaram nenhuma pontuação para o critério.

Tabela 2 - ICMS Socioambiental para o critério "Unidades de Conservação" versus despesas na função gestão ambiental para o ano 2010

\begin{tabular}{l|l|l}
\hline \multicolumn{1}{c|}{ MUNICIPIOS } & \multicolumn{1}{c}{$\begin{array}{c}\text { UNIDADES DE } \\
\text { CONSERVAÇÃO }\end{array}$} & \multicolumn{1}{c}{$\begin{array}{c}\text { DESPESA GESTÃO } \\
\text { AMBIENTAL }\end{array}$} \\
\hline ABREU E LIMA & $88.995,54$ & $8.390,23$ \\
\hline AGUA PRETA & $16.480,66$ & $172.851,70$ \\
\hline ALTINHO & $1.648,07$ & NÃO DISPONIVEIS \\
\hline ARARIPINA & $255.450,17$ & $707.935,40$ \\
\hline BARREIROS & $90.643,61$ & $22.500,00$ \\
\hline BELÉM SÁO FRANCISCO & $26.369,05$ & - \\
\hline BETÂNIA & $51.090,03$ & $703.390,93$ \\
\hline BODOCÓ & $192.823,68$ & $18.819,00$ \\
\hline BREJO MADRE DE DEUS & $9.888,39$ & $48.657,20$ \\
\hline BUÍQUE & $1.418 .984,50$ & $80.605,69$ \\
\hline CABO SANTO AGOSTINHO & $174.694,96$ & $236.270,00$ \\
\hline CARUARU & $31.313,25$ & $773.534,74$ \\
\hline CATENDE & $146.677,84$ & $133.489,05$ \\
\hline CEDRO & $59.330,36$ & - \\
\hline ESCADA & $3.296,13$ & $478.377,23$ \\
\hline EXU & $253.802,11$ & - \\
\hline FLORESTA & $34.609,38$ & $4.470,00$ \\
\hline GOIANA & $690.539,49$ & - \\
\hline IBIMIRIM & $1.049 .817,80$ & $699.873,00$ \\
\hline IGARASSU & $295.003,75$ & $583.976,88$ \\
\hline INAJÁ & $18.128,72$ & - \\
\hline & &
\end{tabular}




\begin{tabular}{|c|c|c|}
\hline IPOJUCA & $36.257,44$ & $125.303,55$ \\
\hline IPUBI & $468.050,64$ & NÃO DISPONÍVEL \\
\hline ITAMARACÁ & $2.628 .664,66$ & $209.785,93$ \\
\hline ITAPISSUMA & $2.658 .329,84$ & $968.108,52$ \\
\hline JABOATÁO GUARARAPES & $118.660,72$ & $845.611,23$ \\
\hline JAQUEIRA & $509.252,28$ & - \\
\hline LAGOA DO OURO & $809.200,22$ & $313.827,44$ \\
\hline LAJEDO & $79.107,15$ & $40.546,50$ \\
\hline MARAIAL & $31.313,25$ & - \\
\hline MOREILÄNDIA & $252.154,04$ & - \\
\hline MORENO & $168.102,69$ & $692.310,06$ \\
\hline OLINDA & $26.369,05$ & $65.108,28$ \\
\hline PAU LISTA & $321.372,80$ & $626.676,30$ \\
\hline PESQUEIRA & $1.648,07$ & $63.066,01$ \\
\hline QUIPAPÁ & $3.296,13$ & $379.722,40$ \\
\hline RECIFE & $196.119,81$ & $11.790 .907,81$ \\
\hline RIO FORMOSO & $565.286,51$ & $2.430,00$ \\
\hline SALOÁ & $14.832,59$ & - \\
\hline SȦO CAETANO & $3.296,13$ & $8.153,00$ \\
\hline SȦO J. COROA GRANDE & $24.720,98$ & $129.506,73$ \\
\hline SAO LOURENQO DA MATA & $303.244,07$ & $196.054,01$ \\
\hline SERRITA & $37.905,51$ & $15.116,48$ \\
\hline SIRINHAÉM & $354.334,11$ & - \\
\hline TACARATU & $13.184,52$ & $238.192,49$ \\
\hline TAMANDARÉ & $665.818,51$ & $205.039,85$ \\
\hline TRINDADE & $3.296,13$ & - \\
\hline TUPANATINGA & $1.267 .362,46$ & - \\
\hline VITORIA DE STO ANTȦO & $8.240,33$ & $212.236,83$ \\
\hline TOTAL DO ESTADO & $16.479 .008,12$ & \\
\hline
\end{tabular}

Fonte: Elaboração dos autores com base nos dados da SEFAZ-PE e do FINBRA

Por meio da análise da tabela 2, com exceção do município de Altinho e Ipubi que não tiveram os dados contábeis disponibilizados, é possível observar que existem municípios que receberam valores do ICMS Socioambiental para o critério unidades de conservação, mas que não apresentara despesa na função gestão ambiental. São eles: Belém do São Francisco, Cedro, Exu, Goiana, Inajá, Jaqueira, Maraial, Moreilândia, Saloá, Sirinhaém, Trindade e Tupanatinga. Outros, como foi o caso do município de Recife, apresentaram despesas superiores aos valores recebidos pela transferência do ICMS. Ressalte-se que não há qualquer obrigação do município em realizar despesas em funções específicas pelo recebimento da transferência do ICMS. 


\section{CONSIDERAÇÕES FINAIS}

A análise dos dados permitiu obter algumas conclusões acerca do instrumento de política pública ICMS Socioambiental no Estado de Pernambuco nos últimos cinco anos no que diz respeito aos critérios unidades de conservação e resíduos sólidos, objetivo deste trabalho.

Primeiramente, ao analisar o Índice de Conservação da Biodiversidade do Município, observa-se que o mesmo vai perdendo desempenho, em sua maioria, na medida em que a análise se desloca dos municípios da Região Metropolitana do Recife ao Sertão. Isso se deve, em alguns casos, ao ritmo mais acelerado de alguns municípios no incremento de novas unidades de conservação que os demais, e em outros casos, por não ter criação de nenhuma unidade de conservação desde o início da política do ICMS Socioambiental, como é o caso do município de Afogados da Ingazeira, por exemplo.

Já em relação à pontuação para resíduos sólidos, a análise permitiu observar que há uma carência muito grande entre os municípios do Estado de Pernambuco, em sua maioria, no adequado tratamento e destinação final de resíduos sólidos, pois apenas 12 municípios apresentaram evolução entre os anos 2008 a 2012 para o critério analisado.

Em relação à análise do perfil das despesas municipais com o meio ambiente versus recursos do ICMS Socioambiental para os critérios resíduos sólidos e unidades de conservação, foi possível observar que 12 municípios apresentaram valores transferidos para o critério unidades de conservação por meio do ICMS, mas que não apresentaram despesas naquele período na função gestão ambiental. Já outros, apresentaram despesas superiores aos valores recebidos pela transferência do ICMS. Isso pode ser explicado pela falta de avaliação qualitativa na prática das unidades de conservação, apesar deste indicador já fazer parte da metodologia de cálculo do ICBM já demonstrada na metodologia deste trabalho.

Por fim, sugere-se que sejam colocadas na prática as avaliações qualitativas para as unidades de conservação de forma que o incentivo à conservação da biodiversidade se 
torne mais eficiente e um maior aprofundamento deste estudo para avaliar as causas da queda de desempenho na maioria dos municípios na medida em que se afastam da Região Metropolitana do Recife em direção ao Sertão.

\section{REFERÊNCIAS}

BRASIL. Constituição Federal do Brasil, artigo 158 de 05 de outubro de 1988. Trata das repartições das receitas tributárias pertencentes aos municípios. Disponível em < http://www.planalto.gov.br/ccivil 03/constituicao/constitui\%C3\%A7ao.htm> Acesso em 14 dez 2011.

CTNSRF, Secretaria da Receita Federal. . Código Tributário Nacional (2007). Disponível em http://www.receita.fazenda.gov.br/legislacao/CodTributNaci/ctn.htm Acesso em 17 de jul 2012.

HEMPEL, Wilca Barbosa. A Importância do princípio protetor recebedor para o desenvolvimento ambientalmente sustentável: o caso do Ceará. Dissertação (Mestrado em Desenvolvimento e Meio Ambiente) - PRODEMA - Universidade Federal do Ceará, Fortaleza, 2006.

JATOBÁ, Jorge. El Impuesto sobre circulación de mercaderias y servicios (ICMS) como instrumento económico para la gestión ambiental: el Caso de Brasil. Política Fiscal y médio Ambiente: bases para uma agenda comun. Santiago do Chile: Acquatella, Jean y Barcena, Alicia, Eds. CEPAL, 2005. Disponível em:

<http://www.cepal.org/publicaciones/xml/4/23634/CapituloV.pdf>. Acesso em: 13 jun. 2008.

PEDROSA, Ivo V. . Crise ambiental: é possível obter contribuição dos governos locais para minimizá-la? Um exame do caso de Pernambuco. In: Quem planeja o território? Atores, arenas e estratégias, 2011, Rio de Janeiro. Quem planeja o território? Atores, arenas e estratégias. Rio de Janeiro : Anpur, 2011.

PERNAMBUCO. Decreto $n^{\circ} 25.574$, de 25 de Junho de 2003. Dispõe sobre a participação das unidades de conservação prevista no art. $2^{\circ}$ da Lei $n^{\circ} 10.489$, d e 02 de outubro de 1990, com a redação da Lei $n^{\circ} 12.206$, de 20 de maio de 2002, na distribuição da parte do ICMS socioambiental que cabe aos Municípios. Disponível em : <http://www.cprh.pe.gov.br/ctudo-secoes sub.asp?idsecao=114\&idlegislacao=413>. Acesso em: 16 jul. 2008. 